\title{
Phenytoin Sodium
}

National Cancer Institute

\section{Source}

National Cancer Institute. Phenytoin Sodium. NCI Thesaurus. Code C48011.

The sodium salt form of phenytoin, a hydantoin derivate and non-sedative antiepileptic agent with anticonvulsant activity. Phenytoin sodium promotes sodium efflux from neurons located in the motor cortex, thereby stabilizing the neuron and inhibiting synaptic transmission. This leads to a reduction in posttetanic potentiation at synapses, an inhibition of repetitive firing of action potentials and ultimately inhibits the spread of seizure activity. 